\title{
Generating Rules for Advanced Fuzzy Resolution Mechanism to Diagnosis Heart Disease
}

\author{
A.V Senthil Kumar, PhD \\ Department of MCA, \\ Hindusthan College of Arts \& Science, Behind Nava India, \\ Coimbatore - 641028. Tamil Nadu, India
}

\begin{abstract}
Fuzzy logic plays an important role in the field of medicine. Many diseases are diagnosed using fuzzy logic. Heart disease is the number one killer to the human community throughout the world. This study was conducted to diagnosis the heart disease among the patients. The components of this study are Fuzzification, Generating rules for Advanced Fuzzy Resolution Mechanism and defuzzification. Crisp values are transformed into fuzzy values through the fuzzification. Generating rules for Advanced Fuzzy Resolution Mechanism has five layers, each layer has its own nodes. In layer 1 rule are generated with the data to frame the new rules and output parameter are predicted. The proposed algorithm is tested with Cleveland heart disease dataset. Generating rules for Advanced Fuzzy Resolution Mechanism was developed using MATLAB Fuzzy Logic Tool Box. Transformation of fuzzy set into crisp values is called Defuzzification. The proposed algorithm can work more efficiently to diagnosis heart disease and also compared with earlier method using accuracy as metrics.
\end{abstract}

\section{Keywords}

Generating rules for Advanced Fuzzy Resolution Mechanism, Rules, fuzzy predicted value, Heart disease

\section{INTRODUCTION}

In Generating rules for Advanced Fuzzy Resolution Mechanism there are number layers with nodes which are connected through links. The Generating rules for Advanced Fuzzy Resolution Mechanism designed with predictive values and if then rules are generated to diagnosis the heart disease.

Detlef Nauck et al.[6] discussed a neuro-fuzzy approach, were learning strategies are used to derive rules from data and results are achieved. Harsh Bhasin and Supreet Singh[10] used genetic algorithms to generate rules in expert system. The rules are generated using correlation coefficient, results are analyzed and it is encouraging. Humar Kahramanli et al.[11] A hybrid model was developed using artificial neural network and fuzzy neural network for diagnosis of diabetes and heart diseases. Li-Xin Wang [14] developed a new method to generate fuzzy rules from the numerical data for time series prediction problem. J-S.R Jang[12] designed fuzzy inference system using adaptive neuro-fuzzy inference system and NN. Mohamad forouzanfar et al.[16] to estimate blood pressure adaptive neuro fuzzy inference system was developed. Min Liu et al. [19] a new ANFIS was designed to predict the parameter of numeric and categorical inputs. MinYou Chen and D.A. Linkens[20] designed a fuzzy rule based model from the numerical data. In this model the fuzzy rules are extracted from the data. The method derived helps to remove the redundant fuzzy rules from the model. Mukhopadhyay et al.[21] accuracy rates are given as $83.04 \%$ and $84.04 \%$ for SVM and GSVM, respectively with Cleveland heart disease database using a new learning model called Granular support vector machines. Robert J. Hammell II[22] developed a fuzzy model, fuzzy rule are derived through training data set. The research presents the architecture of fuzzy model and inference that learns the rule from training dataset. Tarig Faisal et al.[23] designed an Adaptive Neuro-Fuzzy Inference System using subtractive clustering technique to diagnosis dengue patients.

The configuration of this paper is as follows: Section 2 deals with the Adaptive Neuro-Fuzzy Inference System for Heart Disease. The experimental results, implemented in MATLAB fuzzy logic toolbox are presented in Section 3 and experimental result indicates that the proposed Generating rules for Advanced Fuzzy Resolution Mechanism can work more effectively than other methods can [1],[2][3] [7], [9] and [11]in section 4 .

\section{DESIGN OF ADAPTIVE NEURO- FUZZY INFERENCE SYSTEM (ANFIS) FOR HEART DISEASE}

This section describes a Fuzzification, Generating rules for Advanced Fuzzy Resolution Mechanism and Defuzzification as shown in figure 1 .

\subsection{Cleveland heart disease dataset}

To test the ANFIS Cleveland dataset is taken. The Cleveland dataset is retrieved from http://archive.ics.uci.edu/ml and it contains the collected personal data. Table 1 lists the attributes of Cleveland dataset

\subsection{Fuzzification}

Crisp input values are transferred into fuzzy values in the stage of fuzzification [18]. The Fuzzy values are taken into the Generating rules for Advanced Fuzzy Resolution Mechanism. 


\begin{tabular}{|l|l|}
\hline Abbreviation & Full name \\
\hline age & age in years \\
\hline sex & sex $(1=$ male; $0=$ female $)$ \\
\hline cp & chest pain type \\
\hline trestbps & resting blood pressure $(\mathrm{in} \mathrm{mm} \mathrm{Hg})$ \\
\hline chol & serum cholestoral in $\mathrm{mg} / \mathrm{dl}$ \\
\hline fbs & fasting blood sugar $>120 \mathrm{mg} / \mathrm{dl}$ \\
\hline restecg & resting electrocardiographic results \\
\hline thalach & maximum heart rate achieved \\
\hline exang & exercise induced angina \\
\hline oldpeak & ST depression induced by exercise \\
\hline slope & the slope of the peak exercise ST segment \\
\hline ca & number of major vessels \\
\hline thal & $\begin{array}{l}3=\text { normal; } 6=\text { fixed defect; } 7=\text { reversable } \\
\text { defect }\end{array}$ \\
\hline $\begin{array}{l}\text { num } \\
\text { the predicted } \\
\text { attribute }\end{array}$ & $\begin{array}{l}\text { diagnosis of heart disease (angiographic disease } \\
\text { status) }\end{array}$ \\
\hline \multicolumn{2}{|l}{} \\
\end{tabular}

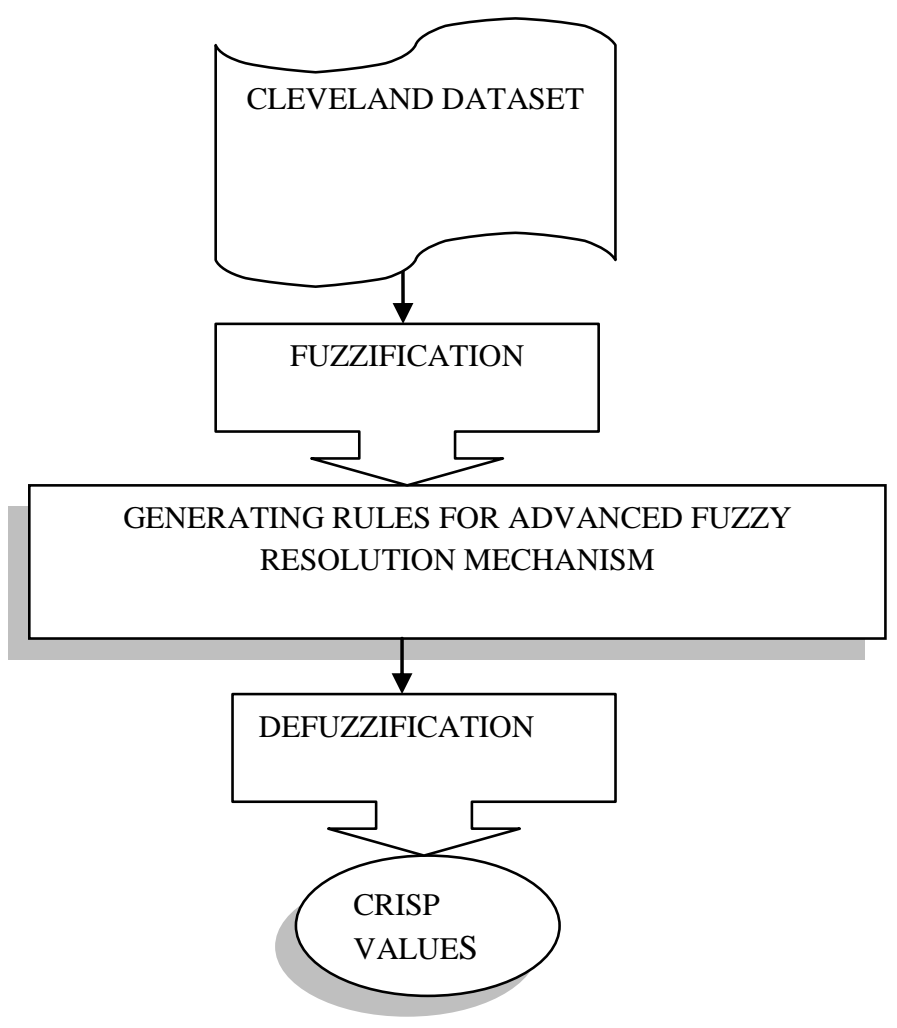

Fig 1: Architecture of Adaptive Neuro-Fuzzy Inference System for Heart Disease

\subsection{An Overview of Generating rules for Advanced Fuzzy Resolution Mechanism for Heart Disease}

Input variables for Generating rules for Advanced Fuzzy Resolution Mechanism are taken from Cleveland dataset. Fourteen variables such as age, sex, cp, trestbps, chol, fbs, restecg, thalach, exang, oldpeak, slope, ca, thal are selected as the input variables and num as output variable.

The first layer uses Sugeno fuzzy model, the output in this model is predicted by fuzzy predicted values. The membership function, fuzzy predicted values; generating if then rules are computed from the Cleveland dataset. The Architecture of the Generating rules for Advanced Fuzzy Resolution Mechanism using ANFIS is shown in figure 2. The parameters are fixed by represented the circular nodes whereas parameters which are to be learnt are represented by square nodes. The fuzzy variables are represented in Table 2 .

\section{Layer 1}

In Layer 1 the node function is the membership of fuzzy set with its related input. Rule based structure is given by first order sugeno fuzzy model [4]. The fuzzy if then rules have input variables, membership function and output variable which are generated from the numerical data. The parameters are determined by Gaussian membership function[17]

$$
O_{i}^{1,3}=\mu_{c p}(x)=e^{-\frac{1}{2}\left(\frac{x-c_{i}{ }^{1}}{\sigma_{i}{ }^{1}}\right)^{2}}
$$

where $\mathrm{c}$ and $\sigma$ represent the membership function center and width respectively in order to determine coordinates of Gaussian membership function.

Table 2. Representation of Fuzzy variables

\begin{tabular}{|l|l|}
\hline Fuzzy variables & $\begin{array}{l}\text { Representation of Fuzzy } \\
\text { Variables }\end{array}$ \\
\hline age & $\mathrm{x} 1$ \\
\hline sex & $\mathrm{x} 2$ \\
\hline cp & $\mathrm{x} 3$ \\
\hline trestbps & $\mathrm{x} 4$ \\
\hline chol & $\mathrm{x} 5$ \\
\hline fbs & $\mathrm{x} 6$ \\
\hline restecg & $\mathrm{x} 7$ \\
\hline thalach & $\mathrm{x} 8$ \\
\hline exang & $\mathrm{x} 9$ \\
\hline oldpeak & $\mathrm{x} 10$ \\
\hline slope & $\mathrm{x} 11$ \\
\hline ca & $\mathrm{x} 12$ \\
\hline thal & $\mathrm{x} 13$ \\
\hline $\begin{array}{l}\text { num the } \\
\text { attribute }\end{array}$ & $\mathrm{y}$ \\
\hline
\end{tabular}

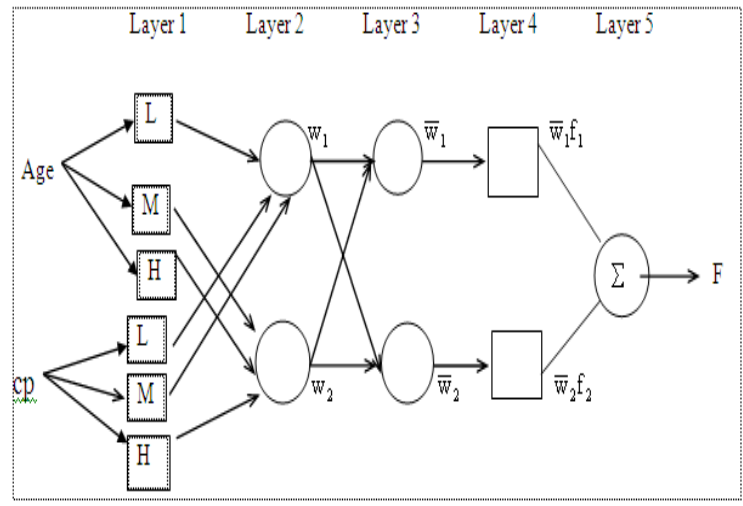

Fig 2: Architecture of the Generating rules for Advanced Fuzzy Resolution Mechanism 


\section{Generating rules using numerical data}

Step 1: The number of data in the database $=191$

Step 2: Calculate the number of attributes $=14$

Step 3: Total number of rules

$=$ Calculate the number of data in the database

Calculate the number of attributes.

$$
=191
$$

$$
=14(\text { approximately })
$$

14

Step 4: Data are sorted according to age and given in Table 3.

Step 5: The maximum value are calculated for each variable.

Step 6: The fuzzy numbers in Table 3 forms the antecedents and consequent part of the rule.

Step 7: Rule 1 is framed as

If ( $\mathrm{x} 1$ is young) or ( $\mathrm{x} 2$ is male) or ( $\mathrm{x} 3$ is high) or ( $\mathrm{x} 4$ is high) or (x5 is medium) or ( $\mathrm{x} 6$ is low) or ( $\mathrm{x} 7$ is high) or (x8 is high) or (x9 is high) or (x10 is high) or (x11 is low) or (x12 is high) or (x13 is high) then (y is high)

Step 8: To frame the next rule, the next 14 data are taken, the step 4, 5 are repeated and generated rules are shown in figure 3

Output variable are determined by predicted fuzzy values for sugeno fuzzy model.Predicted fuzzy values are determined with mean and maximum values for the input and the output variables. The number of attributes for used in predicted fuzzy values is thirteen.

Output variable is predicted using the linear equation $\mathrm{y}=\mathrm{a} 1 \mathrm{x} 1+\mathrm{a} 2 \times 2+\mathrm{a} 3 \times 3+\mathrm{a} 4 \times 4+\mathrm{a} 5 \times 5+\mathrm{a} 6 \mathrm{x} 6+\mathrm{a} 7 \times 7+$ $a 8 \times 8+a 9 \times 9+a 10 \times 10+a 11 \times 11+a 12 \times 12+a 13 \times 13+c$ formula

Parameter is predicted for linear equation using the

$\mathrm{ai}=\frac{\{(\operatorname{mean}(\mathrm{y}) / \operatorname{Max}(\mathrm{xi}))+(\operatorname{mean}(\mathrm{y}) / \text { mean }(\mathrm{xi}))\}}{\mathrm{-mean}(\mathrm{y}) * \text { no. of attributes }}$

The predicted fuzzy values and generated rules are used in ANFIS method to diagnosis heart disease.

\section{Layer 2}

To calculate the firing strength of the rule, nodes are fixed.to T-norm operator with AND operator [19]. The output is derived by the product of all incoming values. Inputs from the nodes in the Layer 1 are multiplied with Layer 2 and the firing strength of the rules is generated. The output of the Layer 2 is given by

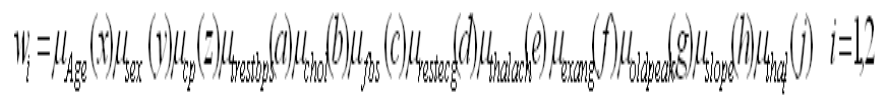

Where $w_{i}$ is the firing strength of rule $i$.

\section{Layer 3}

In this layer nodes calculates the weight, which are normalized. The ith node calculates the portion of the ith rules firing strength to the sum of all rules firing strengths.

$$
=\bar{w}_{i}=\frac{w_{i}}{\sum_{i=1}^{m} w_{i}}
$$

where the output are called normalized firing strengths is of this layer.

\section{Layer 4}

The output of this layer is given with the linear combination of input multiplied by the normalized firing strength. The consequent of the rules are performed in this layer.

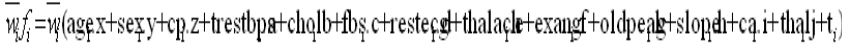

$$
\text { where } \bar{w} \text { is a normalized firing strength from }
$$

layer 3 and

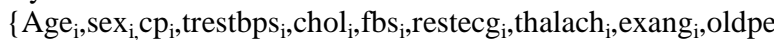
$\mathrm{ak}_{\mathrm{i}}$, slope $_{\mathrm{i}}, \mathrm{ca}_{\mathrm{i}}$, thal $\left._{\mathrm{i}}, \mathrm{t}_{\mathrm{i}}\right\}$ are the parameter set of this node.

\section{Layer 5}

This layer is the simple summation of overall output.

$$
\sum_{i} \overline{w_{i}} f_{i}=\frac{\sum_{i} w_{i} f_{i}}{\sum_{i} w_{i}}
$$

The input is fed through layer by layer. 
Table 3. Numerical data to generate Rule 1

\begin{tabular}{|c|c|c|c|c|c|c|c|c|c|c|c|c|c|}
\hline $\mathrm{x1}$ & $\mathbf{x 2}$ & x3 & $x 4$ & $\mathrm{x5}$ & x6 & $\mathbf{x 7}$ & x8 & x9 & $\mathbf{x 1 0}$ & x11 & $\mathrm{x12}$ & x13 & $\mathbf{y}$ \\
\hline 34 & 0 & 2 & 118 & 210 & 0 & 0 & 192 & 0 & 0.7 & 1 & 0 & 3 & 0 \\
\hline 35 & 1 & 2 & 122 & 192 & 0 & 0 & 174 & 0 & 0 & 1 & 0 & 3 & 0 \\
\hline 37 & 1 & 3 & 130 & 250 & 0 & 0 & 187 & 0 & 3.5 & 3 & 0 & 3 & 0 \\
\hline 37 & 0 & 3 & 120 & 215 & 0 & 0 & 170 & 0 & 0 & 1 & 0 & 3 & 0 \\
\hline 38 & 1 & 1 & 120 & 231 & 0 & 0 & 182 & 1 & 3.8 & 2 & 0 & 7 & 4 \\
\hline 39 & 0 & 3 & 94 & 199 & 0 & 0 & 179 & 0 & 0 & 1 & 0 & 3 & 0 \\
\hline 39 & 0 & 3 & 138 & 220 & 0 & 0 & 152 & 0 & 0 & 2 & 0 & 3 & 0 \\
\hline 40 & 1 & 4 & 110 & 167 & 0 & 2 & 114 & 1 & 2 & 2 & 0 & 7 & 3 \\
\hline 40 & 1 & 1 & 140 & 199 & 0 & 0 & 178 & 1 & 1.4 & 1 & 0 & 7 & 0 \\
\hline 40 & 1 & 4 & 152 & 223 & 0 & 0 & 181 & 0 & 0 & 1 & 0 & 7 & 1 \\
\hline 41 & 0 & 2 & 130 & 204 & 0 & 2 & 172 & 0 & 1.4 & 1 & 0 & 3 & 0 \\
\hline 41 & 0 & 2 & 105 & 198 & 0 & 0 & 168 & 0 & 0 & 1 & 1 & 3 & 0 \\
\hline 41 & 1 & 4 & 110 & 172 & 0 & 2 & 158 & 0 & 0 & 1 & 0 & 7 & 1 \\
\hline 41 & 1 & 3 & 112 & 250 & 0 & 0 & 179 & 0 & 0 & 1 & 0 & 3 & 0 \\
\hline \multicolumn{14}{|c|}{ Maximum values for the fuzzy variables } \\
\hline 41 & 1 & 4 & 152 & 250 & $\mathbf{0}$ & 2 & 192 & 1 & 3.8 & 3 & 1 & 7 & 4 \\
\hline young & male & high & high & medium & low & high & high & high & high & high & low & high & high \\
\hline
\end{tabular}

1. If ( $x 1$ is young) or ( $x 2$ is male) or ( $x 3$ is high) or ( $x 4$ is high) or ( $x 5$ is medium) or ( $x 6$ is low) or ( $x 7$ is high) or ( $x 8$ is high) or ( $x 9$ is high) or ( $x 10$ is medium) or ( $x 11$ is high) or ( $x 13$ is low) or ( $x 13$ is high) then ( $y$ is low) (1) 2. If $(x 1$ is young) or ( $x 2$ is female) or ( $x 3$ is high) or ( $x 4$ is high) or ( $x 5$ is medium) or ( $x 6$ is high) or ( $x 7$ is high) or ( $x 8$ is low) or ( $x 9$ is low) or ( $x 10$ is medium) or ( $x 11$ is high) or ( $x 13$ is medium) or ( $x 13$ is high) then ( $y$ is low) (1) 3. If ( $x 1$ is young) or ( $x 2$ is male) or ( $x 3$ is high) or ( $x 4$ is high) or ( $x 5$ is medium) or ( $x 6$ is low) or ( $x 7$ is high) or ( $x 8$ is high) or $(x 9$ is high) or ( $x 10$ is high) or ( $x 11$ is high) or ( $x 13$ is high) or ( $x 13$ is high) then ( $(y$ is medium) (1) 4. If ( $x 1$ is medium) or ( $x 2$ is female) or ( $x 3$ is high) or ( $x 4$ is high) or ( $x 5$ is medium) or ( $x 6$ is high) or $(x 7$ is high) or ( $x 8$ is high) or ( $x 9$ is low) or ( $x 10$ is high) or ( $x 11$ is high) or ( $x 13$ is medium) or ( $x 13$ is high) then ( $y$ is medium) (1) 5. If ( $x 1$ is medium) or ( $x 2$ is male) or ( $x 3$ is high) or ( $x 4$ is high) or ( $x 5$ is medium) or ( $x 6$ is low) or ( $x 7$ is high) or ( $x 8$ is high) or ( $x 9$ is high) or ( $x 10$ is high) or ( $x 11$ is medium) or ( $x 13$ is high) or ( $x 13$ is high) then ( $y$ is high) (1) 6. If ( $x 1$ is medium) or ( $x 2$ is female) or ( $x 3$ is high) or ( $x 4$ is high) or ( $x 5$ is medium) or ( $x 6$ is high) or ( $x 7$ is high) or ( $x 8$ is high) or ( $x 9$ is low) or ( $x 10$ is high) or ( $x 11$ is medium) or ( $x 13$ is high) or ( $x 13$ is high) then ( $y$ is medium) (1) 7. If ( $x 1$ is medium) or ( $x 2$ is male) or ( $x 3$ is high) or ( $x 4$ is high) or ( $x 5$ is medium) or ( $x 6$ is low) or ( $x 7$ is high) or ( $x 8$ is high) or ( $x 9$ is high) or ( $x 10$ is high) or ( $x 11$ is medium) or ( $x 13$ is low) or ( $x 13$ is high) then ( $y$ is low) (1) 8. If $(x 1$ is old $)$ or $(x 2$ is female) or ( $x 3$ is high) or ( $x 4$ is high) or ( $x 5$ is high) or ( $x 6$ is high) or ( $x 7$ is high) or ( $x 8$ is high) or ( $x 9$ is high) or ( $x 10$ is high) or ( $x 11$ is medium) or ( $x 13$ is high) or ( $x 13$ is high) then ( $(x$ is high) (1)

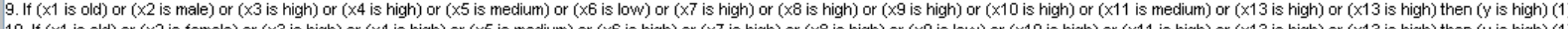

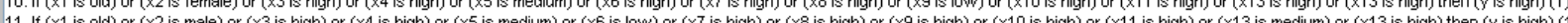
12. If ( $x 1$ is old) or ( $x 2$ is male) or ( $x 3$ is high) or ( $x 4$ is high) or ( $x 5$ is medium) or ( $x 6$ is low) or $(x 7$ is high) or ( $x 8$ is high) or ( $x 9$ is high) or ( $x 10$ is high) or $(x 11$ is high) or ( $x 13$ is medium) or ( $x 13$ is high) then ( $y$ is high) (1) 13. If $(x 1$ is old ) or ( $x 2$ is male) or ( $x 3$ is high) or ( $x 4$ is high) or $(x 5$ is high) or ( $x 6$ is low w or ( $x 7$ is high) or $(x 8$ is high) or ( $x 9$ is high) or ( $x 10$ is high) or ( $(x 11$ is high) or $(x 13$ is high) or $(x 13$ is high) then ( $(y$ is high) (1) 14. If ( $x 1$ is old) or ( $x 2$ is female) or ( $x 3$ is high) or ( $x 4$ is high) or ( $x 5$ is high) or ( $x 6$ is high) or ( $x 7$ is high) or ( $x 8$ is high) or ( $x 9$ is low) or ( $x 10$ is high) or ( $x 11$ is medium) or ( $x 13$ is high) or ( $x 13$ is high) then ( $y$ is medium) (1)

Fig 3: Rule generated from numerical data

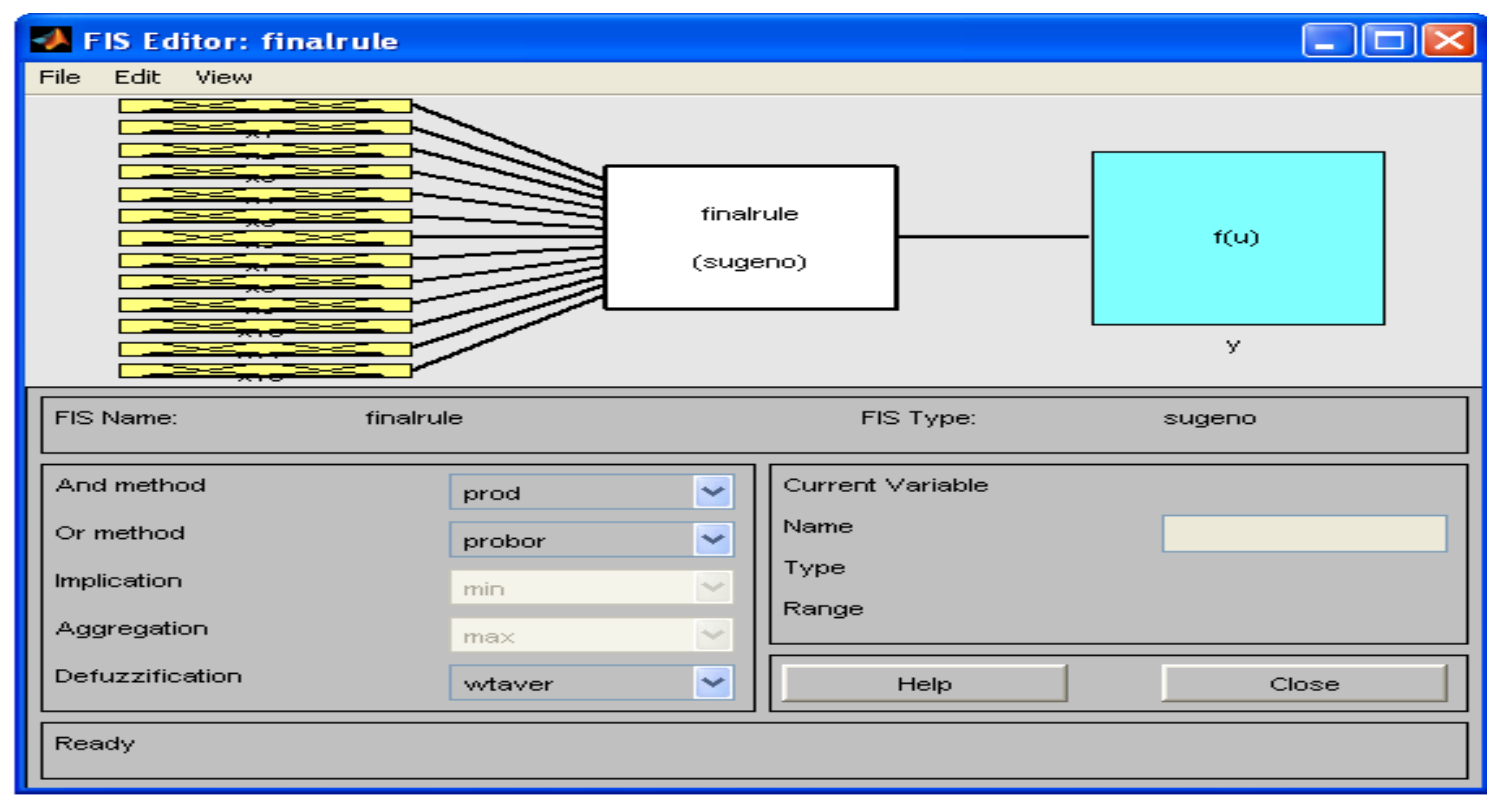

Fig 4: ANFIS modeling framework 


\section{Algorithm for Advanced Fuzzy Resolution Mechanism}

Input

Input the fuzzy set are

$\mathrm{x} 1, \mathrm{x} 2, \mathrm{x} 3, \mathrm{x} 4, \mathrm{x} 5, \mathrm{x} 6, \mathrm{x} 7, \mathrm{x} 8, \mathrm{x} 9, \mathrm{x} 10, \mathrm{x} 11, \mathrm{x} 12, \mathrm{x} 13$.

\section{OUTPUT}

Output the fuzzy set be $y$

\section{Method}

Begin

Step1:Input the crisp values for $\mathrm{x} 1, \mathrm{x} 2, \mathrm{x} 3, \mathrm{x} 4, \mathrm{x} 5, \mathrm{x} 6, \mathrm{x} 7, \mathrm{x} 8, \mathrm{x} 9, \mathrm{x} 10, \mathrm{x} 11, \mathrm{x} 12, \mathrm{x} 13$.

Step 2: Assign fuzzy numbers for the input each variables.

Step 3: Set Sugeno fuzzy model,

Step 4: Layer 1 Call (Generating Rules from numerical data)

Output variable is predicted using the linear equation $\mathrm{y}=\mathrm{a} 1 \mathrm{x} 1+\mathrm{a} 2 \times 2+\mathrm{a} 3 \times 3+\mathrm{a} 4 \mathrm{x} 4+\mathrm{a} 5 \times 5+\mathrm{a} 6 \mathrm{x} 6+\mathrm{a} 7 \mathrm{x} 7+\mathrm{a} 8 \mathrm{x} 8+$ $\mathrm{a} 9 \times 9+\mathrm{a} 10 \times 10+\mathrm{a} 11 \times 11+\mathrm{a} 12 \times 12+\mathrm{a} 13 \times 13+\mathrm{c}$ formula

Parameter is predicted for linear equation using the

$$
\{(\operatorname{mean}(\mathrm{y}) / \operatorname{Max}(\mathrm{xi}))+(\text { mean }(\mathrm{y}) / \text { mean }(\mathrm{xi}))\}
$$

$\mathrm{ai}=$ $\mathrm{i}=1$ to 13

mean $(\mathrm{y}) *$ no. of attributes

Calculate the membership values using triangular membership function.

$$
\begin{aligned}
& O_{i}^{1,1}=\mu_{\text {age }}(x), \text { for }-1,2,3 \\
& O_{i}^{1,2}=\mu_{\text {sex }}(x), \text { for } i-1,2 \\
& O_{i}^{1,3}=\mu_{c p}(x), \text { for }-1,2,3 \\
& O_{i}^{1,4}=\mu_{\text {trestbps }}(x), \text { for } i-1,2,3 \\
& O_{i}^{1,5}=\mu_{\text {chol }}(x), \text { for }-1,2,3 \\
& O_{i}^{1,6}=\mu_{\text {fbs }}(x), \text { for } i-1,2 \\
& O_{i}^{1,7}=\mu_{\text {restec }}(x), \text { for } i-1,2,3 \\
& O_{i}^{1,8}=\mu_{\text {thalach }}(x), \text { for } i-1,2,3 \\
& O_{i}^{1,9}=\mu_{\text {exang }}(x), \text { for }-1,2 \\
& O_{i}^{1,10}=\mu_{\text {oldpeak }}(x), \text { for } i-1,2,3 \\
& O_{i}^{1,11}=\mu_{\text {slope }}(x), \text { for } i-1,2,3 \\
& O_{i}^{1,12}=\mu_{\text {ca }}(x), \text { for } i-1,2,3 \\
& O_{i}^{1,13}=\mu_{\text {thal }}(x), \text { for } i-1,2,3
\end{aligned}
$$

Where $\mathrm{x}$ is input to node and Age,sex.cp,trestbps,chol,fbs, restecg,thalach, exang,oldpeak,slo pe,ca and thal are label in this node.

Step 5: Layer 2 involves fuzzy operators, it uses AND operator to fuzzify the inputs. Layer 2, multiplies the inputs from the nodes in layer 1 and generates the firing strength of the rules.
International Journal of Computer Applications (0975 - 8887)

Volume 77 - No.11, September 2013 Step 6: In Layer 3 the $\mathrm{i}^{\text {th }}$ node calculates the portion of the $\mathrm{i}^{\text {th }}$ rules firing strength to the sum of all rules firing strengths.

$$
=\bar{w}_{i}=\frac{w_{i}}{\sum_{i=1}^{m} w_{i}}
$$

Step 7: In Layer 4, the consequent of the rules are performed by the nodes in this layer.

Step 8: In layer 5 single node computes the overall output:

$$
\sum_{i} \overline{w_{i}} f_{i}=\frac{\sum_{i} w_{i} f_{i}}{\sum_{i} w_{i}}
$$

Step 9: Present the knowledge in the form of human natural language.

End

\section{Generating Rules from numerical data}

Step 1: Calculate the number of data in the database

Step 2: Calculate the number of attributes.

Step 3: Total number of rules to be framed

$=$ Calculate the number of data in the database

Calculate the number of attributes.

Step 4: Sort the data with respect to age

Step 5: Take first 14 data to frame rule 1 , next 14 for rule $2 . .$. Last fourteen for rule 14

Step 6: In first fourteen data take maximum value of the input and output parameters.

Step 7: Set the value for each parameter with the fuzzy numbers.

Step 8: With the fuzzy numbers the rules are framed. of the rules

Step 9: Repeat the step 6, 7 and 8 to frame the rest

\subsection{Defuzzification}

To convert fuzzy values into crisp values defuzzification weighted average method is used. This process is to convert aggregation result into crisp values for the output variable $\operatorname{num}(\mathrm{y})$

\section{EXPERIMENTAL RESULTS}

Generating rules for Advanced Fuzzy Resolution Mechanism was implemented with MATLAB Fuzzy Logic Toolbox. Cleveland dataset was taken to evaluate the performance of the proposed approach. ANFIS modeling framework to diagnosis heart disease is shown in figure 4 . The first experiment shows the membership function for input variable $\mathrm{cp}(\mathrm{x} 3)$ in figure 5 and output variable num(y) in figure 6 . The result of the proposed method is shown in Figure 7. 


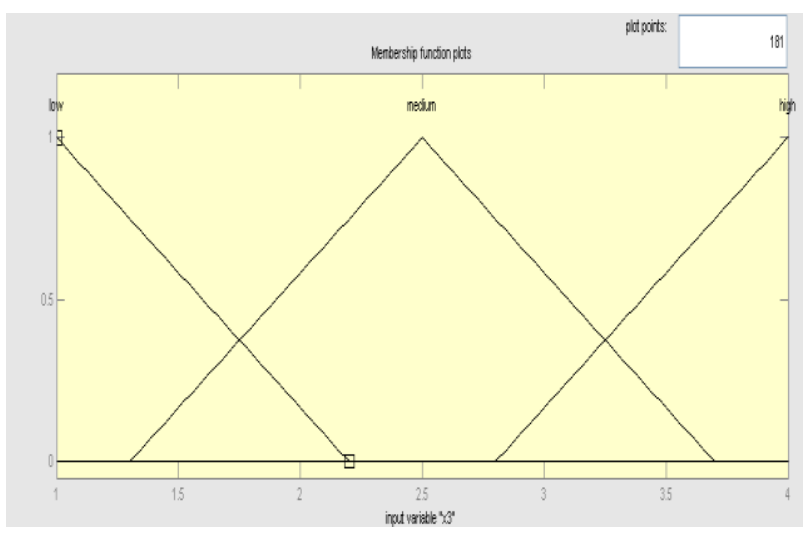

Fig 5: Membership function for input variable $c p(x 3)$

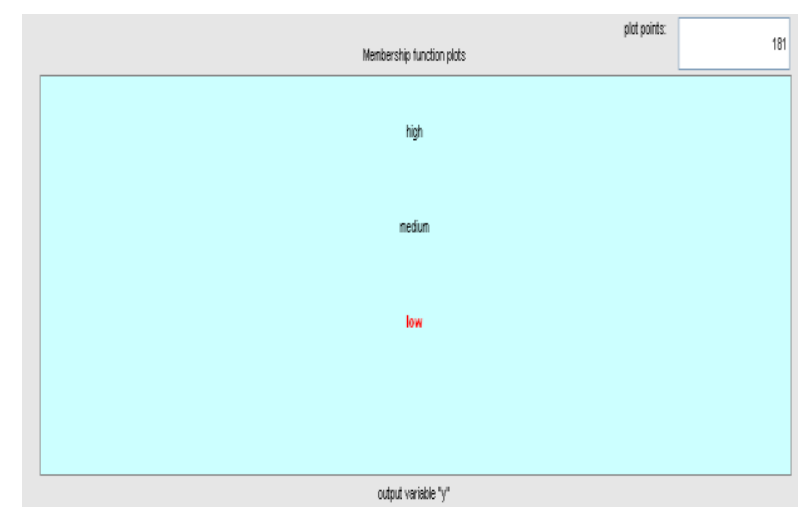

Fig 6: Membership function for output variable $\operatorname{num}(\mathbf{y})$

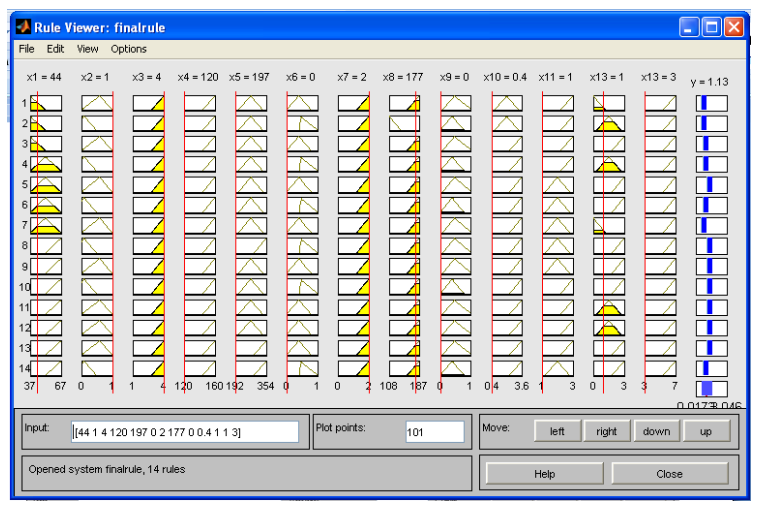

Fig 7: Result obtained from MATLAB

In proposed mechanism fuzzy predicted value technique and rules are used to diagnosis the heart disease. The decision regarding the presence of heart disease can be taken from the figure 7 about the status of angiographic disease.

\section{EVALUATION OF SYSTEM PERFORMANCE}

The performance of the system is evaluated in this stage. Accuracy is metrics used in medical diagnosis. The measure of ability to produce accurate heart diagnosis is determined by accuracy.

So that accuracy [15] is given by eqn. (4)

Accuracy $=$ Total number of correctly diagnosed cases

Total number of cases
International Journal of Computer Applications (0975 - 8887)

Volume 77 - No.11, September 2013

The experimental results are compared with earlier methods involving Cleveland heart disease dataset[1],[2][3] [7], [9] and [11]. Comparing these methods, as listed in Table 4, reveals that the proposed method achieves the first highest accuracy values based on the proposed Generating rules for Advanced Fuzzy Resolution Mechanism. The accuracy values of the proposed method are compared with the earlier methods and represented graphically figure 8 , which shows better accuracy

Table 4. Comparison of Proposed method Accuracy with earlier methods

\begin{tabular}{|c|c|c|}
\hline Method & $\begin{array}{c}\text { Accuracy } \\
(\%)\end{array}$ & Author \\
\hline Current study & 94.11 & $\begin{array}{l}\text { Dr. A.V.Senthil } \\
\text { Kumar }\end{array}$ \\
\hline $\begin{array}{ll}\text { Diagnosis of heart } & \text { disease } \\
\text { using Advanced } & \text { Fuzzy } \\
\text { resolution Mechanism } & \\
\end{array}$ & 93.88 & $\begin{array}{l}\text { Dr. A.V.Senthil } \\
\text { Kumar }\end{array}$ \\
\hline 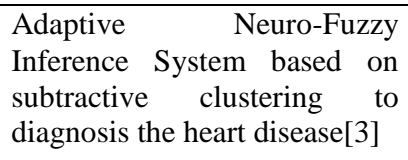 & 92.00 & $\begin{array}{l}\text { Dr. A.V.Senthil } \\
\text { Kumar }\end{array}$ \\
\hline $\begin{array}{l}\text { Diagnosis Of Heart Disease } \\
\text { Using Fuzzy Resolution[2] } \\
\text { Mechanism }\end{array}$ & 91.83 & $\begin{array}{l}\text { Dr. A.V.Senthil } \\
\text { Kumar }\end{array}$ \\
\hline $\begin{array}{l}\text { Adaptive Neuro-Fuzzy } \\
\text { Inference System for Heart } \\
\text { Disease diagnosis [1] }\end{array}$ & 91.18 & $\begin{array}{l}\text { Dr. A.V.Senthil } \\
\text { Kumar }\end{array}$ \\
\hline IncNet[13] & 90 & Norbert Jankowski \\
\hline Hybrid system[11] & 86.8 & $\begin{array}{l}\text { Humar Kahramanli } \\
\text { and Novruz } \\
\text { Allahverdi }\end{array}$ \\
\hline $\begin{array}{l}\text { 26-NN, Manhattan, } 1 \text { feature } \\
\text { removed[9] }\end{array}$ & 86.8 & $\mathrm{WD} / \mathrm{KG}$ \\
\hline 24-NN, Manhattan[9] & 84.8 & WD/KG \\
\hline LDA [5] & 84.5 & Ster and Dobnikar \\
\hline $\begin{array}{l}\text { Fisher discriminant analysis } \\
\text { [5] }\end{array}$ & 84.2 & Ster and Dobnika \\
\hline $\begin{array}{l}\text { FSM, } 82.4-84 \% \text { on test } \\
\text { only[7] }\end{array}$ & 84.0 & Rafał Adamczak \\
\hline Naive Bayes[5] & 83.4 & Ster, Dobnikar \\
\hline $7-\mathrm{NN}[8]$ & 83.2 & $\begin{array}{l}\text { Duch W, } \\
\text { Grudzinski K and } \\
\text { Diercksen G.H.F }\end{array}$ \\
\hline $\mathrm{k}=$ & 82.8 & Rafał Adamczak \\
\hline
\end{tabular}

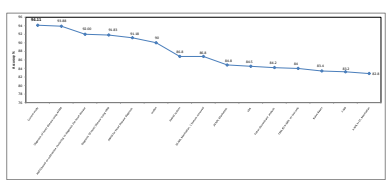

Fig 8: Graphical represent of accuracy 


\section{CONCLUSIONS AND FUTURE RESEARCH}

Rules are generated for Advanced Fuzzy Resolution Mechanism to diagnosis the heart disease. In Cleveland heart disease dataset the values are crisp, converted into fuzzy values by fuzzification process. Generating rules for Advanced Fuzzy Resolution Mechanism has five layers. The rules are generated using numerical data and output variables for the fuzzy model are predicted using fuzzy predicted values to improve the accuracy of the result. The outputs from the Generating rules for Advanced Fuzzy Resolution Mechanism are fuzzy values. Defuzzification process converts fuzzy values into crisp values to known the angiographic disease status. The proposed method has better performance compared with the previous study to diagnosis heart disease. Future Research should test the other related data sets to evaluate its ability to produce a similar accuracy.

\section{REFERENCE}

[1] A.V.Senthil Kumar, "Adaptive Neuro-Fuzzy Inference System for Heart Disease Diagnosis",International Conference on Information System, Computer Engineering \& Application (ICISCEA 2011), Singapore, pp. 91-99,2011.

[2] A.V.Senthil Kumar, " Diagnosis of Heart Disease using Fuzzy Resolution Mechanism”, Journal of Artificial Intelligence 5(1), ISSN 1994-5450, Asian Network for Scientific Information, pp. 47-55, 2012.

[3] A.V.Senthil Kumar, "Adaptive Neuro-Fuzzy Inference System based on substractive clustering to diagnosis the heart disease" , International Journal of Advances in Knowledge Engineering \& Computer Science, Vol.1, Issue. 2, 12 , pp. 01-11. June - 20

[4] Ali Sadighi, and Won-jong Kim, "AdaptiveNeuro-Fuzzy-Based Sensorless Control of a Smart-Material Actuator", IEEE/ASME Transactions on Mechatronics, Vol. 16, No. 2 pp. 374,2011.

[5] B. Ster and A. Dobnikar, ."Neural networks in medical diagnosis: Comparison with other methods". In: A. Bulsari et al., editor, Proceedings of the International Conference EANN '96, pp. 427-430,1996.1

[6] Detlef Nauck, Ulrike Nauck and Rudolf Kruse, "Generating Classification Rules with the Neuro-Fuzzy System NEFCLASS", Proc. Biennial conf. of the North American fuzzy Information Processing Society, pp.1-6,1996.

[7] Duch W, Adamczak R, Grąbczewski K, Żal G, "A hybrid method for extraction of logical rules from data", Second Polish Conference on Theory and Applications of Artificial Intelligence, pp. 61-82,1998.

[8] Duch W, Grudzinski K and Diercksen G.H.F ," Minimal distance neural methods", World Congress of Computational Intelligence, Anchorage, Alaska, IEEE IJCNN'98 Proceedings, pp. 1299-1304,1998.

[9] Duch W, Grudziński K, "A framework for similarity-based methods", Second Polish Conference on Theory and Applications of Artificial Intelligence, pp. 33-60, 1998.
[10] Harsh Bhasin and Supreet Singh, "GACorrelation Based Rule Generation for Expert Systems", Harsh Bhasin et al, International Journal of Computer Science and Information Technologies, Vol. 3 (2), pp. 3733-3736, 2012.

[11] Humar Kahramanli, Novruz Allahverdi,"Design of a hybrid system for the Diabetes and heart diseases", Expert system with applications 35, pp. 82-89, 2008.

[12] J.-S.R. Jang,"ANFIS: adaptive network-based fuzzy inference system", IEEE Trans. Sys. Man. Cybern., vol. 23, pp. 665-685,1993.

[13] Jankowski N, Kadirkamanathan V, "Statistical Control of RBF-like Networks for Classification",7th International Conference on Artificial Neural Networks, Lausanne, Switzerland, pp. 385-390,1997.

[14] Li-Xin Wang, "Generating Fuzzy Rules by Learning from Examples", IEEE Transactions on Systems, Man and Cybernetics, Vol. 22, No.6, pp. 1414-1427,2006.

[15] Loo, C. K., Rao, M.V.C.,"Accurate and reliable diagnosis and classification using probabilistic ensemble simplified fuzzy ARTMAP”, IEEE Transactions on Knowledge and Data Engineering, Volume: 17 Issue:11 pp. $1589-1593,2005$.

[16] M. Forouzanfar, H. R. Dajani, V. Z. Groza, M. Bolic, and S. Rajan," Adaptive neuro-fuzzy inference system for oscillometric blood pressure estimation" in Proc. IEEE Int. Workshop MeMeA, pp. 125-129, 2010.

[17] Marcelo de Carvalho Alves, Edson Ampélio Pozza , João de Cássia do Bonfim Costa, Luiz Gonsaga de Carvalho, Luciana Sanches Alves ,Adaptive neuro-fuzzy inference systems for epidemiological analysis of soybean rust", Environmental Modelling \& Software 26 pp.1089-1096, 2011.

[18] Mehdi Fasanghari, Gholam Ali Montazer,"Design and implementation of fuzzy expert system for Tehran Stock Exchange portfolio recommendation", Expert Systems with Applications 37 pp. 6138-6147, 2010.

[19] Min Liu, Mingyu dong and Cheng Wu," A New ANFIS for Parameter Prediction with Numeric and Categorical Inputs", IEEE Transaction on Automation Science and Engineering Vol. \& No. 3. pp. 645-653, 2010.

[20] Min-You Chen and D.A. Linkens, "Rule-base self-generation and simplification for datadriven fuzzy models", Fuzzy Sets and Systems Vol. 142, pp. 243-265, 2004

[21] Mukhopadhyay, S., Tang, C., Huang, J., Yu, M., \& Palakal, M, "A comparative study of genetic sequence classification algorithms, neural networks for signal processing", In Proceedings of the 2002 12th IEEE workshop, pp. 57-66. 2002. 